\title{
Videotoracoscopía con anestesia peridural en paciente despierto
}

\author{
Epidural anesthesia for videoassisted thoracic \\ surgery in awake patient
}

María Belén Chango Salas ${ }^{1}$ Daysi Noemí Segovia Bustamante ${ }^{1}$, Luis Gangotena Samaniego²

\begin{abstract}
Video-assisted thoracic surgery (VATS) is traditionally performed under general anesthesia and endotracheal intubation with a double lumen tube[1],[2] In recent years, a growing trend towards these procedures being performed under loco regional anesthesia, particularly under epidural block with or without sedation in patients in spontaneous ventilation has appeared. It can be used to perform procedures that include pneumothorax management, wedge resection, lobectomy and surgical reduction of lung volume[1],[3]. The most attractive reason is to eliminate the side effects related to general anesthesia looking for a lower perioperative risks and shorter hospital stays, especially in elderly patients and those with compromised respiratory function[2],[3],[4]. The thoracic epidural anesthesia has been effective allowing an adequate surgical approach, guaranteeing an idoneus level of analgesia, an optimal oxygenation, and facilitating an early postoperative recovery[1]. We present a case of a patient undergoing to lung biopsy performed by VATS patient under epidural block and Ramsay scale sedation level III in spontaneous ventilation, who was discharged 48 hours after the surgical procedure.
\end{abstract}

\section{RESUMEN}

La cirugía torácica asistida por vídeo se realiza tradicionalmente bajo anestesia general e intubación endotraqueal con tubo de doble luz[1],[2]. En los últimos años ha existido una corriente creciente hacia la realización de estos procedimientos en pacientes bajo anestesia locorregional, particularmente con bloqueo epidural con o sin sedación y en ventilación espontánea, para procedimientos

\section{Key words:}

Epidural anesthesia, epidural block, lung biopsy, video assisted, awake

\section{Palabras clave:}

Anestesia epidural, bloqueo epidural, biopsia pulmonar, video asistida, despierto

Médico Posgradista de Anestesiología Universidad San Francisco de Quito, Hospital Carlos Andrade Marín.

Médico Tratante de Anestesiología Hospital Carlos Andrade Marín.

Fecha de recepción: 09 de diciembre de 2018

Fecha de aceptación: 28 de diciembre de 2018

ORCID

https://orcid.org/0000-0002-0746-8867

Correspondencia:

María Belén Chango Salas

Email: mabe_8717@hotmail.com 
que incluyen manejo de neumotórax, resección en cuña, lobectomía y cirugía de reducción de volumen pulmonar[1],[3]. La razón más atractiva es evitar los efectos secundarios relacionados con la anestesia general en búsqueda de menor riesgo perioperatorio y menor estancia hospitalaria, especialmente en pacientes mayores y en aquellos con función respiratoria comprometida[2],[3],[4]. La anestesia epidural torácico (AET) ha sido efectiva para permitir un adecuado abordaje quirúrgico, garantizando un idóneo nivel de anestesia, una correcta oxigenación y facilitando la recuperación posoperatoria precoz[1]. Se presenta el caso clínico de una biopsia pulmonar realizada mediante toracoscopia en un paciente bajo AET con sedación escala Ramsay III y en ventilación espontánea, quien fue dado de alta a las 48 horas posterior a la cirugía.

\section{Introducción}

a anestesia general es la estrategia de primera línea para la cirugía torácica con ventilación unipulmonar, pero incluye riesgos entre los que se describen: traumatismos en dientes o cuerdas vocales, intubación complicada, deterioro de la función cardíaca, inestabilidad hemodinámica, barotrauma alveolar, volutrauma o atelectrauma, atelectasia en el pulmón dependiente secundario al bloqueo neuromuscular, la consecuente derivación de derecha a izquierda y el mayor riesgo de hipoxia intraoperatoria y dependencia del ventilador posoperatorio[5],[6]. Con el uso de técnicas anestésicas locorregionales se reducen los riesgos asociados a la intubación orotraqueal minimizándose la posibilidad de lesión de la vía aérea secundaria al empleo de tubos de doble luz y ventilación unipulmonar. A su vez, la ausencia tanto de relajación diafragmática como de irritación de las vías respiratorias, más una mejor sincronización del movimiento de la caja torácica-abdominal, y la integridad del reflejo tusígeno en el paciente despierto aseguran una mejor ventilación que la obtenida con anestesia general[4].

Se decidió presentar este caso clínico dado la baja frecuencia de la técnica anestésica empleada y los múltiples beneficios de la misma. Entre los últimos encontramos un adecuado colapso pulmonar obtenido mediante el neumotórax quirúrgico, evitar la ventilación mecánica manteniendo la autonomía ventilatoria, minimizar el compromiso hemodinámico y evitar la manipulación de la vía aérea.

\section{Caso clínico}

Paciente masculino de 73 años de edad, con antecedentes de hipertensión arterial, diabetes mellitus tipo 2 e hipotiroidismo que ingresó bajo diagnóstico de nódulos pulmonares en lóbulo medio e inferior derecho. Ingresa para biopsia pulmonar videotoracoscópica por puerto único en segmentos 5 y 7 . Luego de obtener el consentimiento informado por parte del paciente y bajo monitorización básica no invasiva. Previo normas de asepsia y antisepsia, se procede con la ejecución del bloqueo epidural con paciente en posición sentado, sin administración de sedación. Se realiza infiltración local con lidocaína $2 \%$ en la línea media entre las apófisis espinosas de T4 y T5 determinado por reparos de superficie. Luego, con una aguja Tuohy $18 \mathrm{G}$ más técnica de pérdida de resistencia con solución salina 0,9\% $3 \mathrm{ml}$, se identifica el espacio epidural identificado a $4 \mathrm{~cm}$ y se avanza un catéter epidural $20 \mathrm{G}$. Luego de administrar dosis test con lidocaína 2\% + epinefrina 1:2.000.000 $3 \mathrm{ml}$ habiendo descartado posición intratecal o intravascular, se administra bupivacaina sin epinefrina 0,5\% $5 \mathrm{ml}$ + lidocaína $2 \% 5 \mathrm{ml}$ + fentanilo 100 ug, y luego de 10 minutos, se comprueba el nivel sensitivo siendo adecuado. Una vez comprobado el nivel de bloqueo (T3 -T7), se posiciona el paciente en decúbito lateral izquierdo, se mantiene monitorización básica no invasiva: electrocardiograma, presión arterial no invasiva, pulsioximetría, temperatura.

Se aseptiza la piel y se posicionan los campos estériles. El abordaje quirúrgico se realiza mediante una incisión de $4 \mathrm{~cm}$ en sexto espacio intercostal a nivel de la línea axilar anterior, sin reacción que sugiriera dolor ni cambios en frecuencia cardiaca o en la presión arterial, obteniendo un adecuado colapso pulmonar espontáneo. Como primera etapa ante el colapso pulmonar se observa una ligera disminución de Spo2 hasta 94\%, manteniendo respiración paradójica, posterior a lo cual se observa colapso pulmonar total con disminución significativa de Spo2 hasta 86\% misma que fue recuperada y mantenida con administración 
de oxígeno con Fio2 100\% mediante mascarilla facial. Previa manipulación pulmonar se procede a titular sedación con escala Ramsay hasta nivel III administrando midazolam $2 \mathrm{mg}$ iv + propofol $20 \mathrm{mg}$ iv. Durante el procedimiento se mantuvo estabilidad macrohemodinámica y ventilación espontánea con spo2 de $94 \%$ con apoyo de $\mathrm{O}_{2}$ mediante mascarilla facial. Finalizado el procedimiento sin complicaciones, el paciente egresó a la unidad de recuperación. Se mantuvo con analgesia mediante catéter epidural durante 24 horas administrando infusión de Bupivacaína sin epinefrina al 0,0625\% + fentanilo 2,5 microgramos/ $\mathrm{ml}$ velocidad de infusión $6 \mathrm{ml} / \mathrm{h}$. Fue dado de alta a las 48 horas con evidencia clínica e imagenológica de adecuada expansión pulmonar.

\section{Discusión}

La cirugía torácica genera varios cambios fisiológicos especialmente durante procedimientos en pacientes despiertos, no intubados, que requieren ciertas consideraciones[7]. El neumotórax abierto creado quirúrgicamente bajo ventilación espontánea produce la pérdida de la presión negativa pleural de manera que el pulmón afectado se colapsa, generando caída del volumen pulmonar que garantiza adecuado espacio para las maniobras quirúrgicas[1],[3],[4]. El decúbito lateral favorece la ventilación en el pulmón no dependiente y la perfusión en el pulmón dependiente por efecto de la gravedad. El desbalance ventilación perfusión incrementa el riesgo de hipoxemia el cual es menor en pacientes despiertos debido a la contracción eficiente de la hemidiafragma dependiente. En el paciente despierto con ventilación espontánea durante la espiración, el flujo de gas entra en el pulmón afecto proveniente del pulmón dependiente y en inspiración ocurre un fenómeno contrario, lo que se denomina ventilación paradójica. El pulmón no dependiente continúa siendo perfundido, aunque no exista ventilación, lo cual crea un shunt derecha-izquierda intrapulmonar, incrementando el gradiente alveolo arterial de oxígeno produciendo hipoxemia, sin embargo, mecanismos fisiológicos como la vasoconstricción pulmonar hipóxica lo previenen, el cual se encuentra preservado en pacientes con AET[1],[3],[4],[7]. Entre las técnicas de anestesia regional disponibles, algunos procedimientos pueden ser realizados con bloqueos intercostales o bloqueos paravertebrales, los cuales podrían ofrecer muchas de las ventajas de AET, pero con menos efectos secundarios[4]. Efectos cardiovasculares beneficiosos atribuidos a la AET incluyen la disminución de la demanda miocárdica de oxígeno, un mejor flujo sanguíneo miocárdico, mejor función ventricular izquierda, reducción de las complicaciones relacionadas con trombosis y sangrado, reducción de la frecuencia cardíaca y menor incidencia de arritmias intraoperatorias[4],[8]. En el aspecto ventilatorio el mantenimiento del movimiento diafragmático y la presión pleural negativa pueden disminuir los efectos perjudiciales de la presión abdominal sobre el pulmón dependiente. Pompeo et al., demostraron que una hora después de la cirugía se evidenció mejor oxigenación y menor PCO2 en pacientes en quienes el procedimiento se realizó despierto[4],[5].

Entre los beneficios de la anestesia regional se encuentra el bloquear señales neuronales aferentes de tejido quirúrgicamente traumatizado previniendo un aumento de aminomonofosfato cíclico inhibiendo la liberación de catecolaminas. Se ha demostrado que la anestesia local no es tan traumática para el sistema inmune, lo que permite una recuperación más rápida y menor riesgo de infecciones[2],[4]. Inmediatamente después de la cirugía y hasta dos días, la concentración plasmática de cortisol fue menor en el grupo despierto, mientras que la glucosa y la proteína C-reactiva fue más alta en el grupo bajo anestesia general. También se ha determinado que los pacientes sometidos a anestesia locorregional tenían mayor recuento de linfocitos y células natural killer al primer día después de la operación[2],[4]. En cuanto a analgesia, indudablemente AET es superior a la administración de opioides por vía parenteral[4]. Las complicaciones de la AET incluyen hematoma epidural e injuria de la médula espinal, que pueden ocurrir durante la inserción de la aguja debido a trauma directo, nivel de anestesia inadvertidamente alto, incluso con bloqueo del nervio frénico y parálisis hemidiafragmática secundaria. El riesgo predicho de complicaciones neurológicas con AET es $0,07 \%$, en contraste con la anestesia general donde la tasa de complicaciones pulmonares es 33,9\%[4],[5],[6]. Entre los efectos adversos se incluye hipotensión, bradicardia, náuseas y vómitos[4]. La hipotensión puede ser resuelta mediante la administración cuidadosa de fluidos, el soporte vasopresor o inotrópico y evitar la compresión mediastinal excesiva por manipulación quirúrgica[7]. Los inconvenientes de una cirugía VATS despierta son mínimos, el colapso pulmonar total no es posible, ya que los pacientes están respirando espontáneamente, pero los estudios han reportado que el neumotórax abierto inducido por la inserción de los trocares es seguido por un colapso pulmonar casi completo, equivalente al logrado a través de ventilación unipulmonar[2],[3],[9]. En algunos casos se encontró que la viabilidad técnica es menos ideal debido al reflejo tusígeno durante la 
manipulación bronquial, hiperventilación inducida por hipercapnia permisiva y dolor torácico inesperado que requirió anestesia local adicional o sedación[9]. El reflejo tusígeno producto de la manipulación quirúrgica puede ser suprimido con lidocaína intravenosa en concentraciones 1-2 ug/ml, inhalación de lidocaína al $2 \%$ en aerosol con flujo alto de oxígeno 30 minutos antes de la cirugía o bloqueo del ganglio estrellado ipsilateral con $10 \mathrm{ml}$ de bupivacaína al 0,25\%. También se ha descrito que el bloqueo vagal intratorácico con $2 \mathrm{ml}$ de bupivacaína al 0,25\% realizada en algún lugar adyacente al nervio vago ipsilateral podría efectivamente abolir el reflejo tusígeno sin afectar la frecuencia cardíaca, respiratoria o presión arterial[7]. La sedación ligera con infusión continua de Propofol u opioides de vida media corta (remifentanilo) pueden disminuir la frecuencia respiratoria y controlar la ansiedad sin necesidad de conversión a anestesia general, dexmedetomidina también puede ser una opción[4],[6]. Kiss et al., recomiendan que en pacientes con enfermedad pulmonar obstructiva severa se evite sedación o si es necesario usar de manera preferente Ketamina, siendo el único fármaco que mantiene la capacidad residual funcional preservando el tono de los músculos inspiratorios[5]. En general se ha visto que operar bajo anestesia locorregional acorta la estadía hospitalaria, con tiempos de recuperación más rápidos, menor incidencia de complicaciones pulmonares, mayor satisfacción del paciente, menor necesidad de cuidados de enfermería y ahorros de $\operatorname{costos}[2],[4],[8],[9],[10]$. En el caso presentado se demostró que la anestesia epidural y el neumotórax quirúrgico proveen un adecuado campo que permitió realizar el procedimiento sin complicaciones y como medida para evitar el reflejo tusígeno se administró sedación de modo que se aseguró un óptimo campo quirúrgico manteniendo la ventilación espontánea.

\section{Conclusión}

Se pueden realizar de manera eficaz y segura procedimientos toracoscópicos con pacientes despiertos, ventilación espontánea y anestesia epidural torácica con los beneficios de no invadir la vía aérea, sin requerimientos de ventilación mecánica y con estabilidad hemodinámica. El adecuado control analgésico, la menor respuesta inflamatoria y una deambulación precoz son otras de las razones por las cuales la anestesia epidural debe ser considerada en este tipo de procedimientos, permitiendo un alta hospitalaria más rápida.

Conflictos de interés: Ninguno.

\section{Referencias}

1. Alonso-García FJ, Navarro-Martínez J, Gálvez C, Rivera-Cogollos MJ, Sgattoni C, Tarí-Bas IM. Cirugía torácica asistida por vídeo uniportal en paciente despierto. Revista Española de Anestesiología y Reanimación [Internet]. Elsevier BV; 2016 Mar;63(3):17780. http://dx.doi.org/10.1016/j. redar.2015.06.010

2. Klijian AS, Gibbs M, Andonian NT. AVATS: Awake Video Assisted Thoracic Surgery -extended series report. Journal of Cardiothoracic Surgery [Internet]. Springer Nature; 2014 Aug 28;9(1). http://dx.doi. org/10.1186/s13019-014-0149-x

3. Pompeo $E$, Rogliani $P$, Cristino B, Schillaci O, Novelli G, Sal- tini C. Awake Thoracoscopic Biopsy of Interstitial Lung Disease. The Annals of Thoracic Surgery [Internet]. Elsevier BV; 2013 Feb;95(2):445-52. http:// dx.doi.org/10.1016/j.athoracsur.2012.10.043

4. Pompeo E. Awake Thoracic Surgery- Is it Worth the Trouble? Seminars in Thoracic and Cardiovascular Surgery [Internet]. Elsevier BV; 2012 Jun;24(2):10614. http://dx.doi.org/10.1053/j. semtcvs.2012.06.010

5. Kiss G, Claret A, Desbordes J, Porte H. Thoracic epidural anaesthesia for awake thoracic surgery in severely dyspnoeic patients excluded from general anaesthesia. Interactive CardioVascular and Thoracic Surgery [Internet]. Oxford University Press
(OUP); 2014 Jul 17;19(5):816-23. http://dx.doi.org/10.1093/icvts/ ivu230

6. Kiss G, Castillo M. Non-intubated anesthesia in thoracic surgery-technical issues. Ann Transl Med 2015;3(8):109. https:// doi.org/10.3978/j.issn.23055839.2015.05.01

7. Kao M-C, Lan C-H, Huang C-J. Anesthesia for awake videoassisted thoracic surgery. Acta Anaesthesiologica Taiwanica [Internet]. Elsevier BV; 2012 Sep;50(3):126-30. http://dx.doi. org/10.1016/j.aat.2012.08.007

8. Cujiño MD LF, Velásquez MD M. Anestesia peridural con paciente despierto para cirugía de tórax. Revista Colombiana de Neumología [Internet]. Asociación Colombiana de Neumo- 
logía y Cirugía de Tórax; 2017

May 29;24(2):76. http://dx.doi.

org/10.30789/rcneumologia.v24.

$\mathrm{n} 2.2012 .187$

9. Pompeo E, Tacconi F, Mineo D, Mineo TC. The role of awake video-assisted thoracoscopic surgery in spontaneous pneumothorax. The Journal of Thoracic and Cardiovascular Surgery [Internet]. Elsevier BV; 2007 Mar;133(3):786-90. http://dx.doi.org/10.1016/j. jtcvs.2006.11.001

10. Cujiño IF, Velásquez M, Ariza F, Loaiza JH. Anestesia epidural para pleurodesis por toracoscopia: un estudio prospectivo de cohorte. Revista Colombiana de Anestesiología [Internet]. Ovid Technologies (Wolters Kluwer Health); 2013 Jan;41(1):10-5. http://dx.doi.org/10.1016/j. rca.2012.10.003 